\title{
Multiple Nail Gun Penetrating Head Injury: A Case Report
}

\author{
Sim SK, Theophilus SC, Noor Azman AR \\ Department of Neurosurgery, Hospital Sultanah Aminah, 80100 Johor Bahru, Johor
}

\begin{abstract}
Intracranial nail gun injury is a rare subset of penetrating head injury. Here we report a case of intracranial nail gun injury in a Vietnamese patient who attempted suicide with no neurological deficit. Three nails were launched. Because the nail head acted as a brake, the launched nail could make a hole into the skull but could not entirely pass it. A rational management strategy should permit these patients to be discharged with no additional injury. Some medical and surgical management in penetrating head injury are discussed. The use of antibiotics and antiepileptic drugs and the retraction of the nail aided by the performance of a craniotomy surrounding the entry point are recommended.
\end{abstract}

KEY WORDS: Penetrating head injury, nail gun, post-traumatic seizure.

\section{INTRODUCTION}

According to the Center for Disease Control, ${ }^{1}$ the incidence of nail-gun-related injuries presenting to emergency departments in the USA has tripled over the past two decades, with a current annual rate of approximately 37,000 cases with $40 \%$ involving consumers not employed within the construction industry. Although the majority of intracranial nail-gun injuries are work-related accidents, several cases of deliberate self-inflicted harm have been described. ${ }^{2}$

\section{CASE REPORT}

Patient is a-23 year-old Vietnamese factory worker. For some unknown reason, he attempted suicide by shooting himself with a nailing gun at his workplace. Three nails were shot from the right side, and the entry points were one at the right frontal, and two at the right parietal region. There was no loss of consciousness, and he was taken to the local hospital before being referred to our center. Patient's GCS remained full and haemodynamically stable throughout the transportation. No episode of seizure was documented. Broad-spectrum antibiotics and an anticonvulsant were administered on his admission to our center.

On clinical examination, the patient's GCS was E4V5M6, pupils bilaterally equal and reactive. No motor or sensory deficit was found. There was no bladder and bowel involvement as well. Three nail heads were found on the scalp at the right frontal and right parietal region. No other extracranial injury was found.

Corresponding author:

Dr. Sim Sze Kiat

Department of Neurosurgery,

Hospital Sultanah Aminah Johor Bahru,

80110 Johor Bahru, Johor, Malaysia.

Tel: 607-2257000,

Fax: 607-2242694,

Email: ssksimon@gmail.com
Laboratory blood investigations (full blood count, renal profile, and coagulation profile) were within normal limits.

Plain skull radiograph examination in anteroposterior and lateral view revealed three radio opaque foreign bodies on the right frontal and parietal regions. Computed tomography scan of brain revealed the presence of three metallic foreign bodies in the right frontal and parietal regions involving brain parenchyma. One of the foreign bodies at the right parietal region was almost reaching the occipital horn of the right lateral ventricle. No hemorrhagic lesion or associated edema was seen (Figure1).

The patient subsequently underwent craniotomy and removal of the foreign bodies under general anaesthesia. Local scalp incision was made at each entry site of the nails. For each entry point, a burr hole was made adjacent to the nail, and a small circumferential craniotomy was done. The nail was removed cautiously to prevent any vascular insult together with the bone flap. No haematoma was found under the dura. Local cleaning was done with saline and hydrogen peroxide. Fascio-duroplasty was done. The nail was removed from the bone flap. After bactericidal washing, the bone flap was replaced to the craniotomy site, and the skin was closed. All the 3 nails were 2.5 inches long (Figure 2).

Post-operatively the patient recovered well. There was no wound infection or seizure episode. Antibiotics and anti-epileptic medications were completed in one week. The motor and sensory examination revealed normal findings. He was referred to the Psychiatric team for suicidal counseling and it was revealed that he had had an auditory hallucination prior to the incident. The patient was discharged without a neurological deficit on the 7th post-operative day and was given follow-up appointments in the neurosurgical and psychiatric clinics. 


\section{IMJM}

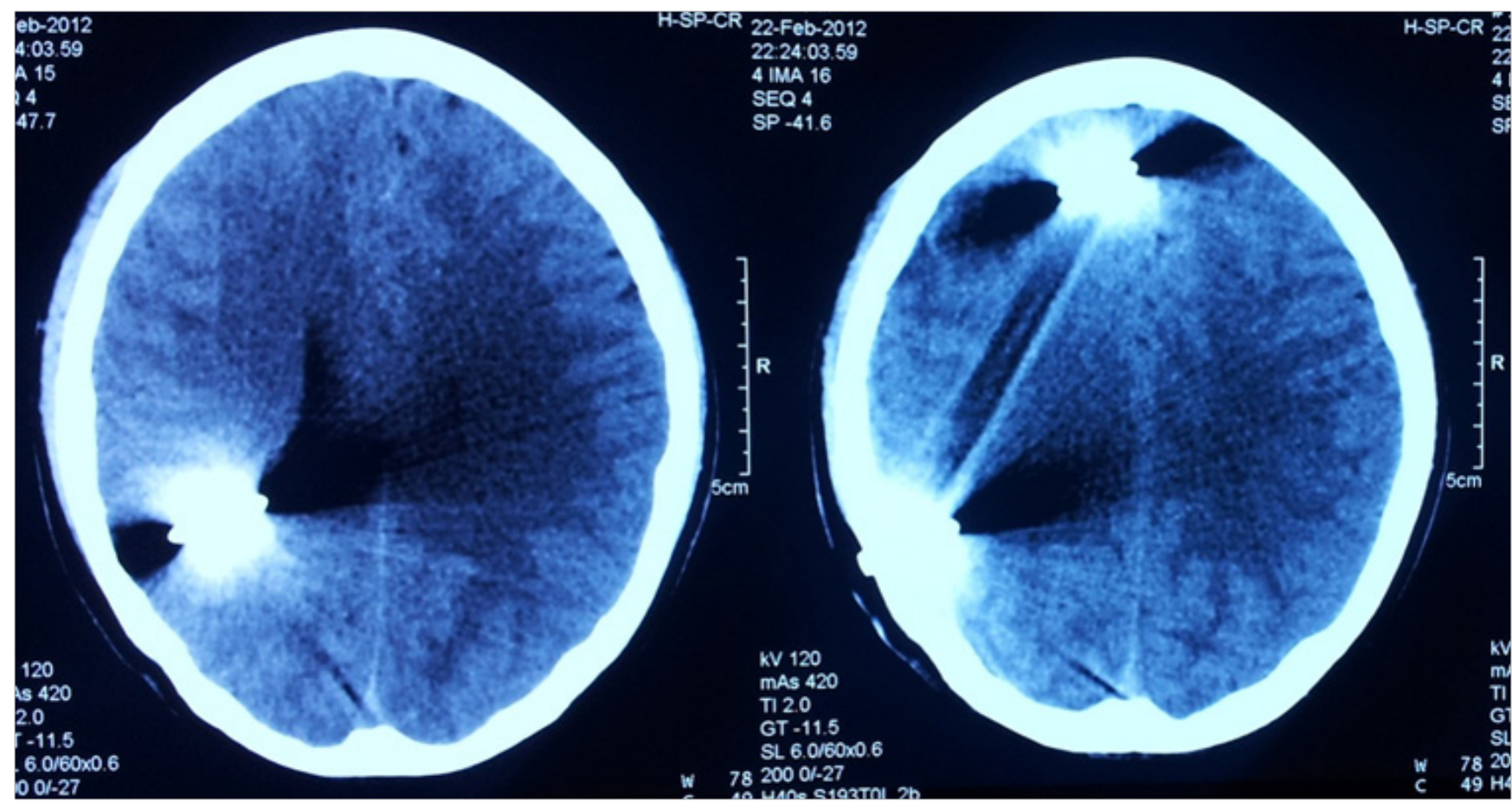

Figure 1. CT brain showed two metallic foreign bodies penetrating the skull and entering the brain matter. No hemorrhagic lesion or associated edema was seen. The third nail was at the high parietal which was not shown in this figure.
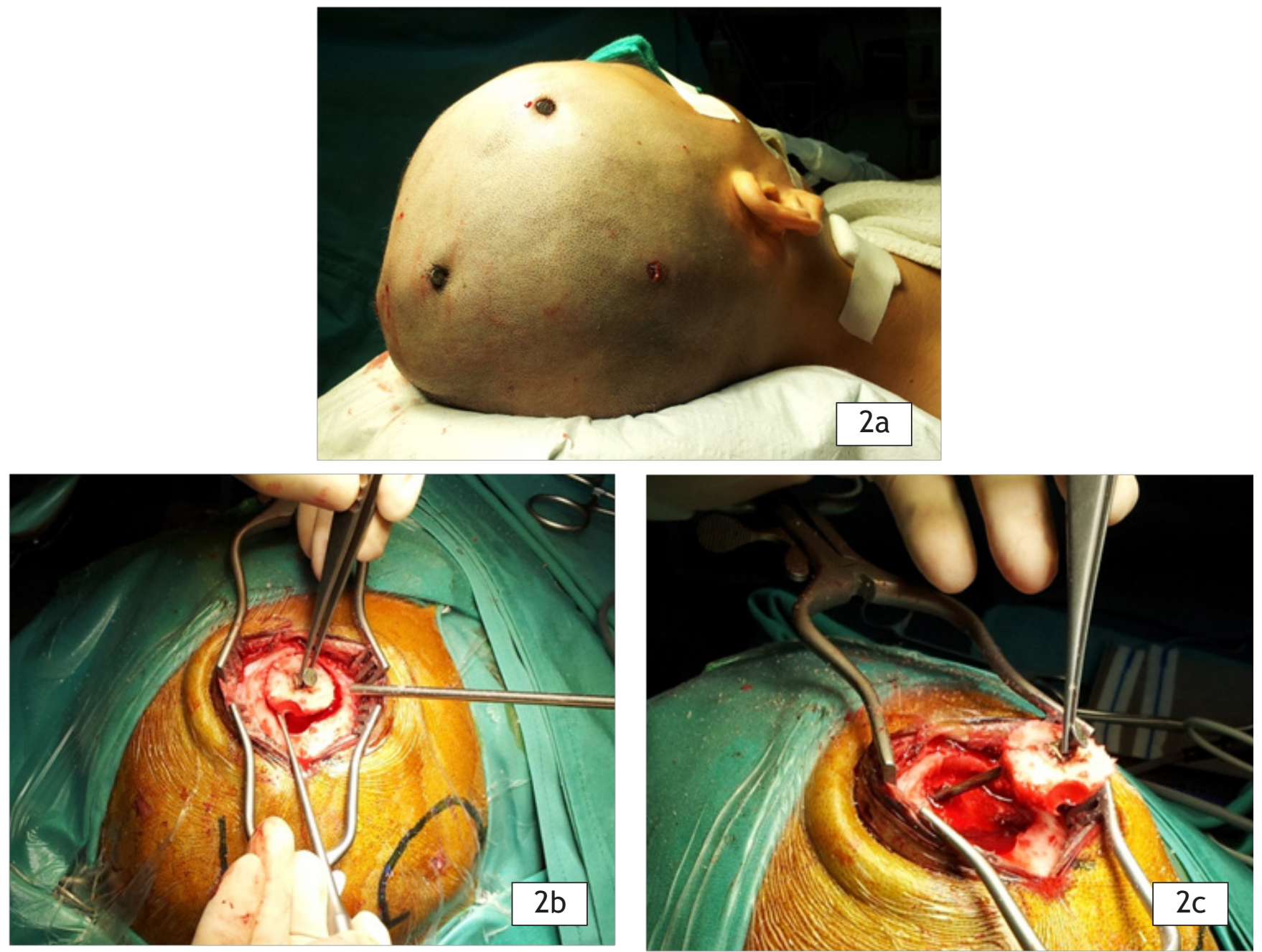

Figure 2.(a) Three nail head were seen on the scalp, one at the right frontal and two at the right parietal regions; (b) local skin incision was made at each entry site of the nail, a burr hole was made adjacent to the nail, and a circumferential craniotomy was done; (c) the nail was removed cautiously to prevent any vascular insult together with the bone flap. 


\section{DISCUSSION}

The most common cause of nail-gun injury is workrelated accidents. ${ }^{1}$ Several cases of deliberate selfharm and suicide using nail-guns have been reported..$^{1,2}$ In most cases, patients survive with a good neurological outcome, provided that the brain stem and the major cerebral vessels are spared. ${ }^{3}$

Neuroimaging is vital in any penetrating head injury for surgical decision making, the type of surgery, the size and site of craniotomy, the route for extraction of the foreign body as well as the decision to choose non-surgical management. CT scanning of the brain is now the primary modality used in the neuroradiologic evaluation of patients with penetrating head injury. This is because CT scanning is quick and provides improved identification of in-driven bone and missile fragments, characterization of the missile trajectory, evaluation of the extent of brain injury, and detection of intracranial hematomas and mass effects. ${ }^{4}$ In addition to the standard axial views with bone and soft-tissue windows, coronal and sagittal sections are also helpful for patients with skull base involvement or high convexity injuries. Postoperatively, CT scan can be helpful in evaluating the development of intracranial hematomas, the presence of residual foreign body, and the extent of cerebral edema.

Cerebral angiography is recommended in patients with penetrating head injury where there is an increased risk of vascular injury. ${ }^{4}$ This would include those cases where the wound's trajectory is through or near the Sylvian fissure (location of $M 1$ and $M 2$ segments of the middle cerebral artery), the supraclinoid carotid artery, the vertebrobasilar vessels, the cavernous sinus region or the major dural venous sinuses. ${ }^{3,4}$ Peripheral branches of the middle cerebral artery followed by the anterior cerebral artery are more vulnerable in craniocerebral penetrating injury than the internal carotid artery. The development of otherwise unexplained subarachnoid hemorrhage $(\mathrm{SAH})$ or delayed hematoma could also suggest the presence of a vascular injury, and thus warrant angiography. ${ }^{4}$ In our case, there was no haemorrhagic lesion seen in the CT scan and clinically patient had no neurological deficit, thus we did not proceed for cerebral angiography evaluation.

Surgical decision and management in penetrating head injuries varies from center to center. In general, more extensive wounds with nonviable scalp, bone, or dura should be debrided more extensively before primary closure or grafting to secure a watertight wound. However, penetrating objects that are of low velocity and of a small calibre such as those found in nail-gun injuries have a low risk of scalp damage, bone fractures or intracerebral haemorrhage. ${ }^{2}$ Selvanathan et al. suggested that if angiography is negative for vascular involvement, and the patient is asymptomatic, the nail should be extracted via closed gentle traction. ${ }^{5}$ They reported a series of patients with intracranial nail-gun injuries successfully removed via closed gentle traction with no haemorrhagic or ischaemic sequelae. It was proposed that intervention involving craniotomy and/ or an endovascular approach should be reserved for those presenting with symptoms of vascular involvement and also when the nail has no extracranial extension. ${ }^{5}$ This is because these invasive interventions may expose patients to unnecessary complications. Nevertheless, there are reports of complications such as subdural haematoma and intraparenchymal haemorrhages following the blind removal of foreign bodies leading to suggestions that all penetrating foreign bodies should be removed under direct vision. ${ }^{6}$ In our patient, mini craniotomies were done at each nailing entry site, and each of the nails was removed together with the bone flap. We think that this method would be safer by reducing the resistance when extracting the nail against the skull bone compared to the closed traction method. In addition, the penetrating wound could be washed thoroughly by removing the nail under general anaesthesia and direct vision, and hence to reduce the risk of infection.

The risk of local wound infections, meningitis, ventriculitis, or cerebral abscess are particularly high among penetrating head injury patients because of the presence of contaminated foreign objects, skin, hair, and bone fragments driven into the brain tissue along the projectile track. Infectious complications are more frequent when there are cerebrospinal fluid leaks, air sinus wounds, and transventricular injuries. ${ }^{8}$

Staphylococcus aureus is the most frequently associated organism. However, gram-negative bacteria also frequently cause intracranial infection after penetrating head injury. ${ }^{8}$ In a large retrospective review of civilian penetrating head injury data, the rate of infection was documented to be $1 \%-5 \%$ with the use of broad-spectrum antibiotics. ${ }^{7}$ There is a considerable variation in the preference for antibiotic regimen for prophylaxis in penetrating head injury patients. The "Infection in Neurosurgery" Working Party of British Society for Antimicrobial Therapy recommended the following regimen for penetrating head injury: intravenous co-amoxiclav 1.2g 8 hourly, or intravenous cefuroxime $1.5 \mathrm{~g}$ first dose, then followed by $750 \mathrm{mg} 8$ hourly with intravenous metronidazole $500 \mathrm{mg} 8$ hourly. It is recommended that this regimen should be started as soon as possible after injury and continued for 5 days postoperatively. ${ }^{8}$ Ideally, surgical treatment should be performed within $12 \mathrm{~h}$ of the injury to decrease the risk of infectious complications. ${ }^{7}$

The risk of post-traumatic epilepsy after penetrating head injury is probably due to direct traumatic injury to the cerebral cortex with subsequent cerebral scarring. The more severe the injury to the brain according to the Glasgow outcome scale grade, the higher the risk for the development of post-traumatic epilepsy. ${ }^{9}$ About $30 \%-50 \%$ of patients suffering a penetrating head injury will develop seizures. It is estimated that up to $10 \%$ of them will appear early (first seven days after the trauma), and $80 \%$ during the first two years, but about $18 \%$ may not have their first seizure until 5 or more years after injury. Recent 
studies recommend the use of prophylactic anticonvulsants (e.g., phenytoin, carbamazepine, valproate, or phenobarbital) in the first week after a penetrating head injury. ${ }^{9}$ The use of anticonvulsants beyond the first 7 days of injury is generally not recommended. In this case, we had continued prophylactic antibiotics and anti-epileptic medications for one week, and there was no infection or posttraumatic epilepsy documented in this patient so far.

\section{CONCLUSION}

This case demonstrates that a nail gun is potentially a dangerous tool in the hands of the inexperienced, especially when a mentally or psychologically disordered personality is involved. A complete preoperative radiologic examination (plain skull films, CTscan), careful surgical removal of the embedded nails by the performance of minicraniotomy surrounding the entry point, the use of antibiotics and antiepileptic drugs and close postoperative neurologic evaluation may prove to be life-saving, even in cases of multiple penetrating craniocerebral injuries caused by a nail gun.

\section{REFERENCES}

1. Center for Disease Control. Nail-gun injuries treated in emergency departments - United States, 2001-2005. Morb Mort Wkly Rep 2007; 56:329-32.

2. Winder MJ, Monteith SJ, Lightfoot N, Mee E. Penetrating head injury from nailguns: a case series from New Zealand. J Clin Neurosci 2008; 15:18-25.

3. Sani S, Jobe KW, Byrne RW. Successful repair of an intracranial nailgun injury involving the parietal region and the superior sagittal sinus. J Neurosurg 2005; 103:567-9.

4. Offiah C, Twigg S. Imaging assessment of penetrating craniocerebral and spinal trauma. Clin Radiol 2009; 64:1146-57.

5. Selvanathan S, Goldschlager T, McMillen J, Campbell S. Penetrating craniocerebral injuries from nail-gun use. J Clin Neurosci 2007; 14: 678-83.

6. Jithoo R, Govender ST, Nathoo N. Penetrating nail gun injury of the head and chest with incidental pericallosal artery aneurysm. S Afr Med J 2001; 91:316-7.

7. Benzel EC, Day WT, Kesterson L, et al. Civilian craniocerebral gunshot wounds. Neurosurgery 1991; 29: 67-71.

8. Bayston R, de Louvois J, Brown EM, et al. Use of antibiotics in penetrating craniocerebral injuries. "Infection in Neurosurgery" Working Party of British Society for Antimicrobial Chemotherapy. Lancet 2000; 355:1813-17.
9. Aarabi B, Taghipour M, Haghnegahdar A, Farokhi MR, Mobley L. Prognostic factors in the occurrence of posttraumatic epilepsy after penetrating head injury suffered during military service. Neurosurg Focus 2000; 8:88-94. 